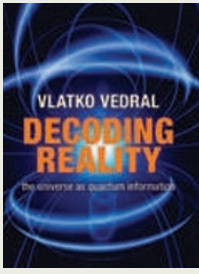

The Universe is an intangible sea of information, suggests physicist Vlatko Vedral in his book Decoding Reality (Oxford Univ.

Press, 2010). Everything around us can be explained through ephemeral statistics. Moreover, much of modern physics, he says, is attributable to the fact that information can be created out of nothing. Vedral challenges our concepts of matter, time, determinism and reality - from the concepts of entropy in thermodynamics to quantum entanglement, and from advances in quantum computing to the Universe itself.

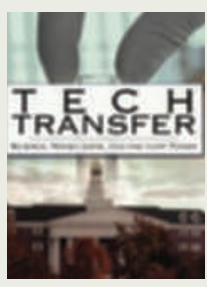

Tech Transfer (CreateSpace, 2010) marks the first foray into fiction for veteran science-policy reporter Daniel Greenberg.

Writing of research life at the mythical Kershaw University, he pokes fun at the machinations of academic institutions. As the book's feuding faculty members plot to disguise their somnolence and to profit from spin-off companies and unethical research, cheating students party on campus. Greenberg's exposé is tongue-incheek and his portrayal harsh, but his messages are serious.

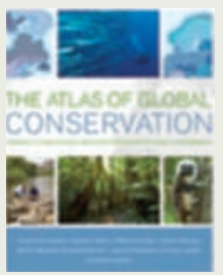

More than 80 richly detailed maps of worldwide environmental data are presented in The Atlas of Global

Conservation (Univ. California Press, 2010). This compilation is written and edited by Jennifer Molnar of The Nature Conservancy and her colleagues, along with journalist Katherine Ellison. The collection highlights the geographical variation of the effects of climate change, water use, habitat protection, deforestation and overfishing, conveyed by sophisticated graphics and informative text. price of one, and paved the way for the modern theory of the 'electroweak' force.

Sample recognizes this work but overlooks its massive counterpart, which is where the excitement lies today. The irony is that it also went largely ignored in 1964. Brout and Englert made no mention of it in their paper, although they were aware of its manifestation in condensedmatter physics. Guralnik, Hagen and Kibble suppressed it in their analysis, which was simplified to focus on the removal of its massless companion. Higgs alone pursued it. What is being called Higgs's boson is, in effect, Goldstone's massive boson. Although at least six physicists can lay claim to this particular mechanism for generating mass, only Higgs realized the importance of the massive boson in testing the theory.

If the above mechanism gives mass to fundamental particles - whether they are the massive $\mathrm{W}$ and $\mathrm{Z}$ bosons; leptons such as the electron or the heavier muon and tau; or even the various flavours of quarks, from lightweight up and down to heavy top, by way of the middleweight charm and bottom - it will be the decay pattern of the Higgs boson into these various particles that will prove it. According to the theory, the Higgs boson will tend to produce the massive flavours of a given family more readily than their lightweight counterparts. It is this pattern that the LHC seeks.

This historical subtlety has given rise to some confusion in nomenclature between the misnamed 'Higgs mechanism' and the Higgs boson itself. The mass-conferring mechanism is the one proposed by the three groups independently, and was anticipated by US physicist Philip Anderson in 1963. The Higgs boson was named by physicists in recognition of Higgs's work on the particle, despite its origins in Goldstone's theory.

To get a sense of the sociology and politics of high-energy physics, Massive is a good place to start. However, if you are assessing competing claims for a Nobel prize, or need to understand the relationship between the widely misattributed Higgs mechanism and the boson that carries his name, be careful.

Frank Close is professor of theoretical physics at the University of Oxford, UK. His forthcoming book on the Higgs boson story, The Infinity Puzzle, will be published next year.

e-mail:f.close1@physics.ox.ac.uk

\title{
Earth-shaking images
}

The Illustrated History of Natural Disasters by Jan Kozák and Vladimir Čermák Springer: 2010. 231 pp. \$89.95, £49.99

Catastrophist ideas have seen a resurgence since the 1980s, when geologist Walter Alvarez associated the dinosaurs' disappearance with an asteroid impact. The sense that cataclysmic events shape the planet has been reinforced by a string of recent natural disasters - the 2004 Indian Ocean earthquake, Hurricane Katrina in 2005 and the 2010 Haiti earthquake. Even minor geophysical events can disrupt populations, as the current Icelandic volcanic ash cloud demonstrates.

The Illustrated History of Natural Disasters, by geophysicists Jan Kozák and Vladimir Čermák, is a timely collection of pictorial records of earthquakes, volcanoes and tsunamis from antiquity to modern times. It shows how such events - and the way they were illustrated influenced the development of geoscience. Accompanied by explanatory text, the images include copper engravings, woodcuts and oil paintings, produced for purposes ranging from scientific observation to newspaper reporting. The result is a hybrid book that intertwines the history of science and art.

The authors focus on Italian volcanoes, which

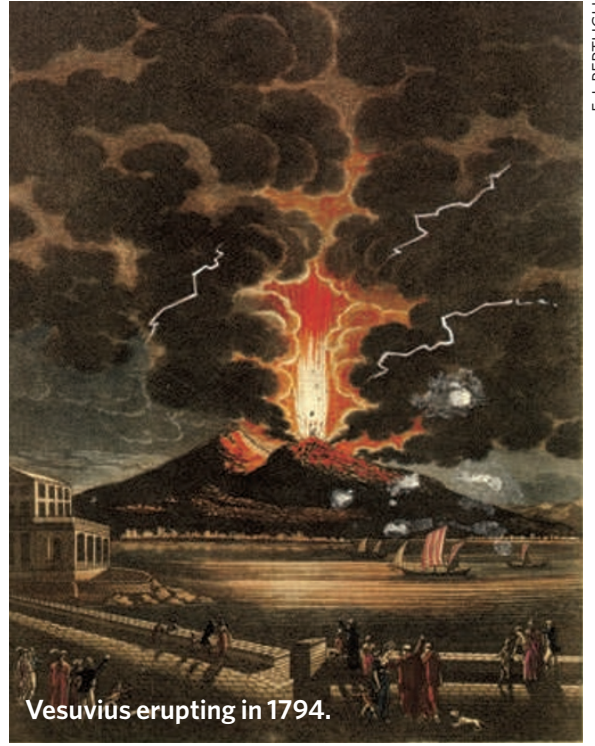

were particularly influential. Mount Vesuvius, which buried in ash the cities of Herculaneum and Pompeii in $79 \mathrm{AD}$, became a natural laboratory for geologists when it resumed activity in the mid-seventeenth century. William Hamilton, the British ambassador who lived in Naples from 1764, devoted himself to studies of Vesuvius, including monitoring the increasing frequency of eruptions during the second half 